\title{
Design of the Monitoring System about Tailing Dam Infiltration Line Based on Zigbee
}

\author{
Guoshao Chen ${ }^{1, a}$ and Zhongsheng Wang ${ }^{2, b}$ \\ ${ }^{1}$ College of Computer Science and Engineering, Xi'an Technological University, Xi'an 710021, \\ P.R.China \\ a1825247141@qq.com, b59483672@qq.com
}

Keywords: Zigbee; wireless sensor network; tailing dam

\begin{abstract}
Tailings is very important facilities in mine enterprise production. Tailings dam safety related to the safety of life and property of the dam downstream residents. Therefore, they pay more attention on the safety of tailings dam. According to the need of tailings safety monitoring, the ZigBee communication technology will be used to dam water level monitor. The system will alarm once the data is beyond the Threshold. Through experiments show that ZigBee based tailings water level monitoring system, with characteristics of low power consumption, convenient construction, are important to the safety of tailing dam.
\end{abstract}

\section{Introduction}

Tailing pond is the place that was usually used to accumulate tailings. Tailing pond in China is widely distributed and present some characters, such as large quantity, small scale, poor stability and a large proportion of dangerous. All above indicate security situation is very grim. Larger proportion of tailings and its fine particles determine that tailings reservoir is not only a dangerous source of man-made debris flow with high potential, but also containing harmful substances, such as sulfide, chloride, cyanide and so on. Once the dam were break, it will cause great harm to the lives and property of downstream residents and serious pollution of the natural and ecological environment, which also has the impact to sustainable economic development and social stability. Therefore, tailings dam plays a major role in the safety of tailing pond. And the detection of the infiltration line is the key of tailing dam detection.

Conventionally, artificial detection is used to detect the infiltration line of tailings dam.Inspectors need regularly measure the depth of monitoring points by means of traditional instrument,however, this manual testing workload is relatively large, and the measurement is easily effected by many factors(such as artificial), which may cause certain system and artificial error. In addition, artificial detection can not gain the infiltration line in real time, it also affect the safety of the tailings dam.

The monitoring system of tailing dam infiltration line based on ZigBee can realize the real-time remote collection of the tailing dam.

This paper mainly introduces the design of the ZigBee based monitoring system of the tailing dam infiltration line, whose data acquisition node is composed of a single chip microcomputer board and a pressure sensor.This system uses radio frequency CC2530 (ZigBee) technology for data transmission and the related software on PC is developed serials of technology,such as C\# and Sql Server. Then, the infiltration line of tailings dam is displayed on the system interface in real time.According to the calculation of infiltration line, the system provides data basis for automatic alarm.

\section{Introduction of ZigBee Technology}

ZigBee is a low-cost, low-power and short-range wireless communication technology.It is suitable for wireless communication with low speed and short distance in almost all industries, besides, it is often applied in the smart home, industrial field data collection, intelligent transportation, intelligent 
building and digital hospital, etc. ZigBee is characterized by low rate, low power consumption, low-cost, self configuration and flexible network topology.Due to low transmission rate of ZigBee, its transmission data is very small ,the time of signal transceiver is short and the relative anti-interference ability is strong.In the non-working mode, ZigBee nodes can automatically fall sleep, so it is very power-saving. Because the ZigBee protocol stack is much simpler than other wireless transmission technology, it reduces the requirement of the calculation of the controller. Therefore, we can use 8 bit microcontroller and small capacity memory to reduce the cost of system development largely. This system adopts CC2530 chip made by TI company, which integrates the 51 core and transmit receive module.Then, only a small amount of peripheral circuit can form a complete wireless transceiver circuit. In the reachable distance, ZigBee automatically establishes the network through coordinators and access to related channel using CSMA-CA.The terminal nodes in ZigBee can join and exit casually, and it is a kind of self-organizing configuration. ZigBee has multiple network topology and it can be flexible accordance with the different needs of the corresponding topology.

\section{The Design of System Structure}

This system consists of three parts, which are data acquisition node, ZigBee wireless communication and database management system .The data acquisition node is responsible for converting the water level of tailings dam detection hole into digital quantity; ZigBee wireless communication system is responsible for the collection of all the data and send it to the host computer; Database management system will receive the data to save, calculate and alarm. Each ZigBee module also has a relay function, so as not to be too far to complete the transmission. The overall system is shown in Fig. 1.

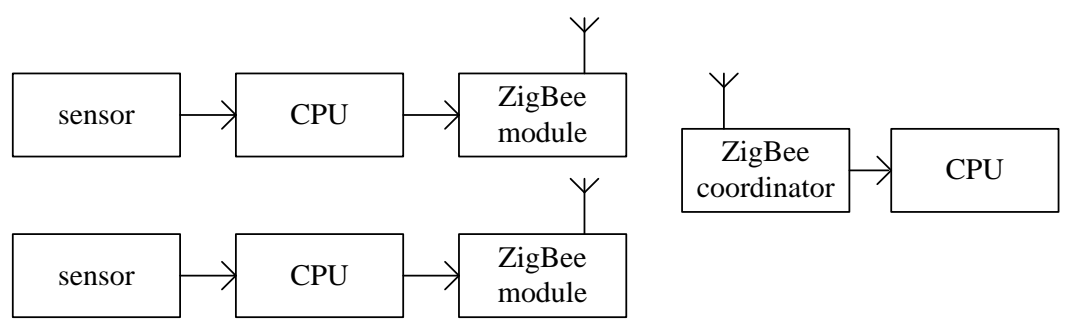

Figure 1. Overall structure of the system

\section{The Structure of System Hardware}

The structure of this monitoring system hardware consists of industrial control computer, data acquisition node and ZigBee communication.The structure of these parts shown in Fig. 1. Coordinator is unique in a ZigBee network, which mainly comprises a microcontroller, a radio frequency transceiver unit, a power supply module and an interface unit. In hardware selection of the system, the accuracy, stability and economic applicability of the system are mainly considered. In ZigBee network, the CC2530 is used as the main processor of communication module, and the data acquisition uses STC12LE5A60S2 MCU as the processor.

The system data acquisition node includes pressure sensor, MCU processing board and ZigBee module. The pressure sensor was put into the water level detection hole according to requirements, then, MCU processing board and ZigBee node were placed in the top of the detection hole.Firstly, the MCU processor converts the signal of pressure sensor into the standard Modbus protocol data and transfer it to ZigBee. Then, the ZigBee module transmits data to the coordinator according to the network protocol. The industrial control computer could receive these data through RS232 serial port and dispose data in database management system. When the data acquisition node is too far 
away from the coordinator, the information can not be transmitted directly to the coordinator.At this time, data can be transferred to other reachable terminal-nodes for relaying, and then transmit it to the coordinator.

\section{Software Design of the System}

The system software comprises two parts: the embedded software part and the database management software.Embedded software mainly completes the task of converting the sensor's current signal into standard Modbus PDU and wireless transmission. Database management software could collect the water level information and store these information in the SERVER SQL database to carry out standardized management.The staff can query infiltration of historical information through the software and set the alarm threshold saturation line. These data can trigger alarm system automatically.

Embedded Software. Embedded software is composed of ZigBee wireless communication software and water level data acquisition software.ZigBee wireless communication software using Ti standard protocol stack provided by the company,then it is not primary question in this paper.The latter Water level data acquisition software is priority.

The data acquisition software flow chart is shown in Fig. 2.First of all,system initializes serials of settings including format and rate of communication, watchdog timer setting, AD conversion module working mode, I/O pin settings etc.Once initialization completes, the system stay a wait status and wait for industrial control. When an instruction is received by its own address, the CRC check process starts.If the result of CRC check is correct, the ADC function begins to work.Eventually,the software could transmit the results of the $\mathrm{AD}$ conversion to the ZigBee wireless communication software.
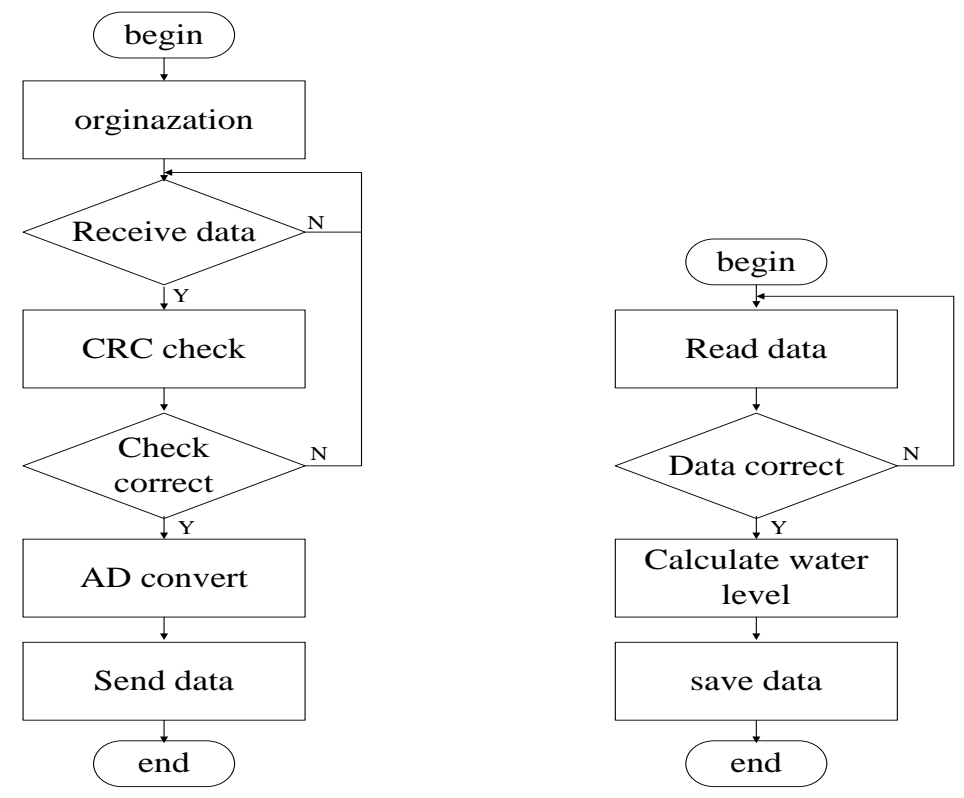

Figure 2. Flow chart of embedded software

The Database Management Software. The data collected by the terminal is transmitted to the coordinator through ZigBee, in order to make the data can be transmitted to the industrial control computer, the serial communication program is necessary, which is relatively simple in this situation and can be realized by calling related controls, modifying the parameters. Then, industrial computer sends data-read instructions and waits for the response of terminal. If the returned data cannot pass CRC check, it is necessary to send read directive again.Otherwise, the water level data 
is extracted and stored to the database.At the same time, comparing stored data with the alarm threshold, if the stored result is exceeded, the alarm function would start. CRC check function is as follows:

//This function provides the preparation for the following calculation of the 16 bit CRC

int calcByte(int crc, char b)

$\{$ int $\mathrm{i}$;

$\mathrm{crc}=\operatorname{crc}^{\wedge}($ int $) \mathrm{b}<<8 ; \quad$ for $(\mathrm{i}=0 ; \mathrm{i}<8 ; \mathrm{i}++)$

$\{$ if $((\operatorname{crc} \& 0 \times 8000)==0 \times 8000)$

$\mathrm{crc}=\mathrm{crc}<<1 \wedge 0 \times 1021$;

else

$\operatorname{crc}=\operatorname{crc}<<1 ;$

\}

return crc \& 0xffff;

\}

int CRC16(char *pBuffer, int length) // the function of 16bit CRC computation

$\{$ int $\mathrm{WCRC} 16=0$;

int $\mathrm{i}$;

for $(\mathrm{i}=0 ; \mathrm{i}<$ length; $\mathrm{i}++$ )

$\{\quad$ wCRC16 = calcByte $($ wCRC16, pBuffer[i] $)$;

\}

return wCRC16;

\}

I/O Module. I/O module plays an important role in database management.It includes staff login management, work parameter settings, historical data query, real-time data display and alarm, etc. The display module of real-time data is the most intuitive module in the whole monitoring system,it could show collected data in the form of real-time curve, which is drawing by the he GDI+ library provided by .NET framework. The data of each collection point in tailing dam would form a tow-dimension graphic,whose horizontal axis is time and vertical is water level.This graphic is refreshed in a fixed time, the real-time changes of water level would be directly displayed on the screen.

\section{Conclusion}

Monitoring system of tailing dam infiltration line based on ZigBee realizes the measurement and calculation of the water level in each monitoring hole of tailings dam.This system has the characteristics of reliable data transmission, simple and flexible networking, low cost, low power consumption and so on,which could collect water level data and save the information of the infiltration line in real time.In this case, staff could timely grasp the health status of tailings dam, so as to realize the safety status of tailings, which could be in line with the current demand of safety production. This system is worthy of application.

\section{References}

[1] Qu Lei, Liu Shengde, Hu Xianbin. Technology and application of ZigBee. Beijing: Beihang University press.

[2] Zhang Shuili Liu Xing. Based on stc12c5a16s2 microcontroller of the rock soil thermal testing instrument design and implementation of.Proceedings of 2010 National Vocational Education of communications and Information Technology Conference (2010 NVCIC) in the "2010.

[3] Zheng Yingren Weimin. Calculation seepage force drawdown and phreatic. Chinese Journal of rock mechanics and Engineering. 